\title{
Interlimb coordination during the stance phase of gait in subjects with stroke
}

Andreia S. P. Sousa (PhD)

Escola Superior da Tecnologia de Saúde do Instituto Politécnico do Porto, Área Científica de Fisioterapia, Centro de Estudos de Movimento e Actividade Humana, Rua Valente Perfeito, 322 - 4400-330 Vila Nova de Gaia, PORTUGAL

Faculdade de Engenharia, Universidade do Porto, Rua Dr. Roberto Frias, s/n, 4200-465 Porto, PORTUGAL

E-mail: asp@estsp.ipp.pt, andreia.asps@gmail.com

Augusta Silva (PhD)

Escola Superior da Tecnologia de Saúde do Instituto Politécnico do Porto, Área Científica de Fisioterapia, Centro de Estudos de Movimento e Actividade Humana, Rua Valente Perfeito, 322 - 4400-330 Vila Nova de Gaia, PORTUGAL

E-mail: smaugusta@gmail.com

Rubim Santos (PhD)

Escola Superior da Tecnologia de Saúde do Porto, Área Científica de Física, Centro de Estudos de Movimento e Actividade Humana, Rua Valente Perfeito, 322 - 4400-330 Vila Nova de Gaia, PORTUGAL

E-mail: rss@estsp.ipp.pt

Filipa Sousa $(P h D)$

Laboratório de Biomecânica do Porto, Faculdade de Desporto, Universidade do Porto, Porto, PORTUGAL

E-mail: filipas@fade.up.pt

(corresponding author) João Manuel R. S. Tavares (PhD)

Instituto de Engenharia Mecânica e Gestão Industrial, Departamento de Engenharia Mecânica, Faculdade de Engenharia, Universidade do Porto, Rua Dr. Roberto Frias, s/n, 4200-465 Porto, PORTUGAL

E-mail: tavares@fe.up.pt, Phone: +351 22508 1487, 


\begin{abstract}
Objective: To analyse the relation between contralesional and ipsilesional limbs in subjects with stroke during step-to-step transition of walking.

Design: Observational, transversal, analytical study with a convenience sample.

Setting: Patients from a physical medicine and rehabilitation clinic in Portugal (Braga).

Participants: Sixteen subjects with post-stroke hemiparesis with the ability to walk independently and twenty-two healthy controls.

Interventions: Not applicable.

Main outcome measures: Bilateral lower limbs electromyographic activity (EMG) of soleus (SOL), gastrocnemius medialis (GM), tibialis anterior (TA), biceps femoris (BF), rectus femoris (RF) and vastus medialis (VM) muscles and ground reaction force were analysed during double support and terminal stance phases of gait.

Results: The propulsive impulse of the contralesional trailing limb was negatively correlated to the braking impulse of the LEAD limb during double support $(r=-0.639$, $\mathrm{p}=0.010$ ). A moderate functional relation was observed between thigh muscles ( $\mathrm{r}=-$ 0.529, $\mathrm{p}=0.035$ ), and a strong and moderate dysfunctional relation was found between the plantar flexors of the ipsilesional limb and the vastus medialis of the contralesional limb, respectively (SOL-VM, r=-0.800, p<0.001; GM-VM, r=-0.655, p=0.002). Also, a functional moderate negative correlation was found between the SOL and RF muscles of the ipsilesional limb during terminal stance and between the SOL ( $\mathrm{r}=-0.506$, $\mathrm{p}=0.046)$ and $\mathrm{VM}(\mathrm{r}=-0.518, \mathrm{p}=0.040)$ muscles of the contralesional limb during loading response, respectively. The trailing limb relative impulse contribution of the contralesional limb was lower than the contribution of the ipsilesional limb of subjects with stroke $(\mathrm{p}=0.02)$ and lower than the relative impulse contribution of the healthy limb $(\mathrm{p}=0.008)$ during double-support.
\end{abstract}

Conclusions: The findings obtained suggest that the lower performance of the contralesional limb in forward propulsion during gait is not only related to contralateral supraspinal damage but also to a dysfunctional influence of the ipsilesional limb.

Keywords: gait energy consumption; step-to-step transition; electromyographic activity; propulsive impulse. 


\section{INTRODUCTION}

Gait disorders affect a large proportion of subjects with stroke, limiting their ability to ambulate in the community ${ }^{1}$. The features of post-stroke walking vary according to stroke severity, location of infarct, time since stroke, type of rehabilitation received and other individual differences ${ }^{2}$. Also, the mechanical energy cost per stride and metabolic energy expenditure ${ }^{3}$ are typically higher in subjects with stroke in comparison to healthy subjects.

Biomechanical models have shown the importance of interlimb relation during the double-support phase (DS) in unimpaired gait energy consumption ${ }^{4}$. Indeed, the transition from one stance limb inverted pendulum to the next appears to be a major determinant of the mechanical work of walking ${ }^{4,5}$. An optimal mechanical relation between human limbs has been described as the trailing limb (TRAIL) plantar flexor action ${ }^{6,7}$ compensates the energy loss provoked by the leading limb (LEAD) during heel strike ${ }^{5,8}$ to maintain the velocity of the body's centre of mass. Recent studies involving healthy subjects have demonstrated that the degree of plantar flexor activity during propulsion depends on the degree of muscle activity ${ }^{9}$ and on the magnitude of the ground reaction force ${ }^{10}$ of the contralateral limb during heel strike. This interlimb relation observed during step-to-step transition of unimpaired walking ${ }^{9,}{ }^{2}$, and also in standing-related tasks ${ }^{11,12}$, can be justified by the bilateral influence of group II fibres on spinal interneurons ${ }^{13}$ and by the importance of vestibulo- and reticulo-spinal pathways on group II fibres ${ }^{14}$.

After a unilateral stroke, interlimb coordination is often impaired ${ }^{15-17}$, it is either caused by the primary brain lesion itself and/or due to adaptive changes ${ }^{18-21}$. Although studies about interlimb relation have been dedicated to the evaluation of upper limbs, neurophysiological and neuroanatomical findings indicate that the interlimb coordination of lower limbs can be impaired, particularly when there are subcortical injuries in the territory of the middle cerebral artery, such as in the internal capsule ${ }^{22,23}$.

Subjects with stroke present low kinetic energy ${ }^{24,25}$ and an inadequate propulsion of the contra-lateral limb to the affected hemisphere (contralesional limb) during preswing ${ }^{26}$ as a result of low plantar flexor strength and power ${ }^{2,27}$. The major metabolic cost has been associated with the mechanical work done by the ipsilesional limb, mainly to lift the centre of mass ${ }^{28}$. However, in spite of often being described as a compensatory limb that adapts to changes in the paretic limb ${ }^{29}$, changes observed in the 
ipsilesional limb have also been attributed to a possible dysfunction of ipsilateral distributed pathways responsible for postural control ${ }^{29-33}$. This could help us to understand why stroke subjects present lower performance of the contralesional limb when cyclic and antiphase ankle movements are executed with both limbs ${ }^{34,35}$. In fact, these findings suggest that the ipsilesional limb may lead to performance changes in the contralesional limb and reinforce the idea that step-to-step transition during gait could be highly demanding in terms of energy consumption in stroke subjects due to their need to coordinate contralesional and ipsilesional limbs.

The main purpose of this study was to analyse the relation between ipsilesional and contralesional limbs during gait step-to-step transition in terms of individual muscle activity and global kinetic values in subjects with stroke. Taking into account the changes observed in both contralesional ${ }^{26,27}$ and ipsilesional ${ }^{30}$ limbs during gait, a dysfunctional interlimb relation was hypothesised, compared to interlimb relation patterns observed in healthy subjects ${ }^{9}$. Specifically, a higher dysfunctional relation would be expected between the ipsilesional heel strike limb and the contralesional propulsion limb. This hypothesis is based on the role of the ipsilateral and contralateral pathways, as the former is more related to postural control, highlighted in the moment of touchdown, and the later are more associated to movement control, highlighted during propulsion ${ }^{22}$.

To the best of our knowledge, no previous study has evaluated the interlimb relation during an asymmetric task implying a supportive role for the two limbs in subjects with stroke. Whereas correlation analyses have revealed that some EMG abnormalities such as spasticity ${ }^{36}$, altered co-contraction ${ }^{37}$, and muscle paresis ${ }^{29}$ are higher in subjects with severe stroke, a cause-effect relationship of some of these abnormalities with poor locomotor performance ${ }^{3}$ remains difficult to establish. The study of interlimb relation during step-to-step transition in subjects with stroke can give significant insights to improve our understanding of the low performance of stroke gait, considering the importance of step-to-step transition performance in global gait efficiency. Restoring gait is one of the major goals in stroke rehabilitation, and therefore understanding the interlimb relation is extremely beneficial for designing effective locomotor interventions.

\section{METHODS}




\subsection{Subjects}

Sixteen (8 females; 8 males) patients who had suffered a stroke at least 6 months earlier and 22 healthy subjects (12 females; 10 males) participated in this study (Table 1). For the subjects with stroke, the mean time between their stroke and the time of inclusion in this study was 26 months ( $S D=9)$. All subjects suffered an ischemic stroke: 11 of them had suffered an infarction in their left hemisphere, whereas 5 had suffered an infarction in their right hemisphere. To be included, patients were required to: (1) have suffered an ischemic first-ever stroke involving the territory of the middle cerebral artery, as revealed by computed tomography, resulting in hemiparesis; (2) have a FuglMeyer (Assessment of Sensorimotor Recovery After Stroke scale) score in the motor subsection below 34; (3) have the ability to walk 10 meters, with close supervision if necessary, but without physical assistance, as judged by the treating physiotherapist; (4) have provided written or verbal informed consent. Patients were excluded for one of the following reasons: (1) cognitive deficit that could hinder communication and cooperation (assessed by the Mini-Mental State Examination); (2) history of orthopaedic or neurological (other than stroke) disorders, known to affect walking performance; (3) history of stroke involving the brainstem or cerebellar areas; and (4) taking medication that could affect motor performance. Given the possibility of spastic hypertonus, an experienced neurological physical therapist assessed all subjects by testing limb resistance to passive movement and considering the definition of spastic hypertonus, all subjects included in the study were considered as not having spastic hypertonus in the lower extremity. Gait data of the group of subjects with stroke were compared with data obtained from the 22 healthy control subjects. All control group subjects were sedentary and were selected according to the same exclusion criteria applied to the stroke group, as well as being excluded if they had suffered any neurological disorder. The study was approved by the local ethics committee and implemented according to the Declaration of Helsinki.

\subsection{Instrumentation}

The values of the vertical $\left(F_{z}\right)$, anteroposterior $\left(F_{y}\right)$ and mediolateral $\left(F_{x}\right)$ components of the ground reaction force (GRF) were acquired using two force plates at a sampling rate of $1000 \mathrm{~Hz}$ (FP4060-10 and FP4060-08 models from Bertec Corporation (USA), connected to a Bertec AM 6300 amplifier and to a Biopac 16-bit 
analogical-digital converter, from BIOPAC Systems, Inc. (USA)). The two force plates were mounted in series near the midpoint of the walkway.

The activity of the agonist muscles for propulsion (Gastrocnemius Medialis (GM), Soleus (SOL), Rectus Femoris $(\mathrm{RF})^{38}$ ) and initial contact and loading response (Tibialis Anterior (TA), Vastus Medialis (VM) and Biceps Femoris $(\mathrm{BF})^{38,}{ }^{39}$ ) was assessed through electromyography (EMG). The bilateral EMG signal of these muscles was monitored using a bioPLUX research wireless signal acquisition system (Plux Ltda, Portugal). The signals were collected at a sampling frequency of $1000 \mathrm{~Hz}$ and were preamplified in each electrode and then fed into a differential amplifier with an adjustable gain setting (25 - $500 \mathrm{~Hz}$; common-mode rejection ratio (CMRR): $110 \mathrm{~dB}$ at $50 \mathrm{~Hz}$, input impedance of $100 \mathrm{M} \Omega$ and gain of 1000). Self-adhesive silver chloride EMG electrodes were used in a bipolar configuration and with a distance of $20 \mathrm{~mm}$ between detection surface centres. The skin impedance was measured with an Electrode Impedance Checker (Noraxon USA, Inc.). The EMG and force platform signals were analysed with the Acqknowledge software (Biopac Systems, Inc., USA). The gait timing was measured using a photovoltaic system (Brower Timing IRD-T175, Utah, USA). All subjects used the same shoe type, in their adequate size.

\subsection{Procedures}

\subsubsection{Skin preparation and placement of electrodes}

The skin surface of selected muscles' mid-belly and patella was prepared (shaved and then the dead skin cells and non-conductor elements were removed with alcohol and with an abrasive pad) to reduce the electrical resistance to less than $5000 \Omega$, the EMG electrodes were placed according to anatomical references, and the reference electrode was placed on the patella.

\subsubsection{Data acquisition}

\section{a) Kinetic and electromyographic data}

The EMG and GRF data were simultaneously acquired during walking. Subjects walked using a standard footwear over a 10 m walkway ${ }^{40}$ without using any assistive devices and/or orthotics.

Before the data acquisition, sufficient time was given so that the participants become familiar with the experimental settings. They were allowed to walk over the 
walkway without explicit instructions. In the meanwhile, we observed the starting point on the walkway from which they placed one foot on the first force plate (TRAIL) and the other on the second force plate (LEAD) according to their natural cadence. To ensure a low intra-group gait variability ${ }^{41-44}$ and a similar rate of energy expenditure ${ }^{3}$ and muscle utilization ratios or levels of effort ${ }^{45,46}$ between the two groups the subjects walked at their self-selected speeds. Three successful trials, where the feet had full contact with the plate, were used for analysis for all subjects to reduce the withinindividual variability and increase statistical power ${ }^{47}$. One minute breaks were provided between trials.

\subsubsection{Data processing}

\section{a) Kinetic parameters}

The GRF data were low-pass filtered using a fourth-ordered Butterworth filter by using a zero-phase lag with a cutoff frequency of $8 \mathrm{~Hz}^{48}$ and normalised according to body weight. In spite of occurring mainly during DS, biomechanical models suggest that energy loss during step-to-step transition can be reduced by a propulsion impulse from the TRAIL limb immediately before collision of the LEAD limb ${ }^{5}$. Also, studies about interlimb relation in healthy subjects demonstrate a relation between muscle activity of the TRAIL limb during terminal stance and biomechanical parameters of the LEAD limb during initial contact and loading response ${ }^{9,10}$. Thus, the stance phase was separated into three intervals (Figure 1) to analyse the impulse generation at various time points in the gait cycle: (1) DS corresponding to the initial contact and loading response of the contralesional limb until the start of the ipsilesional limb swing, (2) terminal stance (TS) of the contralesional and ipsilesional limbs, and (3) DS corresponding to initial contact and loading response of the ipsilesional limb until the start of the contralesional limb swing. Globally, each limb was evaluated during TS, pre-swing and loading response. The stance phase was defined as the interval where $\mathrm{F}_{\mathrm{z}}$ presents a value equal to or higher than $7 \%$ of body weight (BW) ${ }^{41}$, the DS corresponds to the time between the initiation of the LEAD stance phase and the initiation of the TRAIL swing phase, and the TS was defined as the time between the time where $\mathrm{F}_{\mathrm{y}}$ assumes the value zero $\left(\mathrm{F}_{\mathrm{y}}=0\right)$ and the beginning of the second DS (Figure 1). Variables derived from $\mathrm{F}_{\mathrm{y}}$ were time integrated to assess the braking (negative $\mathrm{F}_{\mathrm{y}}$ ) and propulsive (positive $\mathrm{F}_{\mathrm{y}}$ ) impulses during each interval. The percentage of the propulsion impulse 
(\%PI) generated by the TRAIL limb during the DS in relation to the LEAD limb braking impulse was calculated according to the following equation ${ }^{49}$ :

$\% P I=\frac{\int F y_{\text {trail }}}{\int F y_{\text {trail }}+\int F y_{\text {lead }}} \times 100$,

where $\int F y_{\text {trail }}$ is the integral of $F_{y}$ of the TRAIL limb during DS and $\int F y_{\text {lead }}$ is the integral of $F_{y}$ of the LEAD limb during DS. The percentage of propulsive contribution provides a quantitative measure of the coordinated output of each leg for forward propulsion during the DS of walking ${ }^{49}$.

\section{b) Electromyographic activity}

The EMG of both limb muscles was analysed during the intervals selected to evaluate the propulsive and braking impulse (Figure 1). The EMG signals were filtered using a zero-lag, second-order Butterworth filter with an effective band pass of 20-450 $\mathrm{Hz}$, and the root mean square (RMS) was calculated for each interval. The EMG values obtained at each interval were normalised to the mean signal for each muscle over the entire gait cycle ${ }^{50}$. Only the EMG activity of stroke subjects was analysed, as the interlimb relation in terms of EMG activity was analysed in our previous study ${ }^{9}$.

\subsection{Data analysis}

The acquired data was analysed using the Statistic Package Social Science (SPSS) software from IBM Company (USA). Spearman's and Pearson correlation coefficient tests were used to assess the relation between contralesional and ipsilesional limbs in terms of EMG and propulsive/braking impulse, respectively. As already mentioned, in healthy subjects, the interlimb relation was analysed only in terms of propulsive/braking impulse. The Paired Samples T-test was used to compare the propulsive and braking impulse levels and the relative propulsive contribution between contralesional and ipsilesional limbs. To compare the propulsive impulse level between limbs of subjects with stroke and between stroke and control group in the three stance subphases, the Bonferroni correction was used to reduce type I error. Statistical significance was set at $\mathrm{p}<0.05$.

\section{RESULTS}




\subsection{Interlimb relation: EMG activity}

Figure 2 shows that, during DS, the higher values of the ipsilesional GM, SOL and BF activity at initial contact and loading response are associated to lower values of the contralesional VM activity during pre-swing, while during TS higher values of ipsilesional SOL and RF activity are associated to lower values of contralesional SOL and VM activity at initial contact and loading response. It was also interesting to note that the higher the contralesional VM activity was during pre-swing, the higher the RF $(r=0.514, p=0.05)$ and GM activity were $(r=0.539, p=0.038)$ for the same limb.

\subsection{Interlimb relation: propulsive and braking impulse}

Lower values of propulsive impulse and of relative propulsive contribution were found in the contralesional limb of the stroke group during pre-swing, Figures 3 and 4, respectively. In the control group there were statistically significant correlations between the propulsive impulse of the TRAIL limb and the braking impulse of the LEAD limb during DS ( $\mathrm{r}=-0.568, \mathrm{p}=0.004)$; however in the stroke group, only the braking impulse of the ipsilesional LEAD limb was related to the propulsive impulse of the contralesional TRAIL limb during DS ( $\mathrm{r}=-0.639, \mathrm{p}=0.010)$.

\section{DISCUSSION}

Healthy and stroke groups adopted different walking speeds, as they were asked to walk at their own comfortable speeds. The adoption of a low self-selected speed has been shown to provide stroke subjects with a rate of energy expenditure ${ }^{3}$ and muscle use ratios or levels of effort ${ }^{45,46}$ that are similar to those of healthy subjects walking at their comfortable speeds. In this sense, it can be argued that the differences observed in self-selected speed did not interfere with our results.

Each limb has been shown to affect the strength of muscle activation and timespace behaviour of the other ${ }^{9,52}$. The results of our study demonstrate that in subjects with stroke, the EMG and impulse levels of the TRAIL limb were related to the ones of the LEAD limb during DS, but only when the LEAD limb was the ipsilesional limb and the TRAIL limb was the contralesional limb. The higher levels of ipsilesional BF activity were associated with lower levels of the contralesional VM activity, which despite not developing an important role in this subphase is positively correlated to the 
level of activity of the GM that contributes to swing initiation ${ }^{53}$ and of the RF that accelerates the trunk forward ${ }^{38,39}$. Taking into account that the BF muscle action has been related to impact reduction during initial contact and loading response ${ }^{40,54,55}$, the inverted indirect relation established with the GM and RF activity during pre-swing is consistent with the inverted correlation observed between the ipsilesional braking impulse and the contralesional propulsive impulse. Based on the step-to-step transition model prediction ${ }^{5}$ that the TRAIL limb propulsion compensates the energy loss of LEAD limb during initial contact and loading response, these results seem to demonstrate that the ipsilesional limb improves the coordination deficits of the contralesional limb during DS. This is probably because all appropriate ipsilesional sensorimotor information can be integrated by the nervous system and thus contribute to a more appropriate pattern in the contralesional limb. However, it should be noted that the SOL activity increases the horizontal energy of the trunk much more than the GM, especially in the late stance ${ }^{53,56}$, and no influence was exerted by this muscle in the ipsilesional limb.

Moreover, an inverted strong/moderate correlation was also observed between ipsilesional SOL and GM activity during initial contact and loading response and the contralesional VM activity during pre-swing. Considering that the SOL and GM antagonists has an agonist roles in impact reduction ${ }^{57}$, it would be expected, according to the reciprocal inhibition mechanism, that higher SOL and GM values would be associated with higher VM levels. This non-functional interlimb relation could be the result of excessive co-activation values of plantar flexor and dorsiflexor muscles of the ipsilesional limb ${ }^{29}$ as a consequence of ipsilaterally mediated effects from the neurological lesion ${ }^{30,43}$ and/or to an adaptation for poor stability during gait ${ }^{37}$. However, no differences were observed in the ankle muscle co-activation between the ipsilesional limb of subjects with stroke and control subjects (unpublished work). Another explanation could be related to recent evidence indicating that the spinal group II excitation from ankle dorsiflexors to knee extensors is particularly enhanced during post-stroke in initial stance walking ${ }^{58}$. Considering that most midlumbar interneuron recipients from group II input are influenced by afferent fibres from both ipsilateral and contra-lateral sides ${ }^{13}$, a relation from ankle dorsiflexors during initial contact and loading response to contra-lateral knee extensors during pre swing could be expected. However, our results did not support this relation. 
The contralesional limb muscle activity during initial contact and loading response was also correlated to the ipsilesional limb muscle activity during TS. Considering that TS precedes initial contact, the results seem to indicate that higher levels of the ipsilesional limb SOL could potentiate the activity of the contralesional limb TA during loading response through a decrease of SOL activity. This influence is close to the interlimb relation observed in healthy subjects 9 .

When comparing the EMG results obtained in this study with the ones obtained in healthy subjects from a global point of view ${ }^{9}$, it is evident that the TA, BF and VM muscles in the healthy subjects have an important role in contralateral limb activity, while in the stroke subjects the ipsilesional SOL muscle has consistently more influence over the contralesional limb. Besides that, the TA did not have any role in interlimb relation in stroke subjects. The lack of influence that the ipsilesional limb has over the contralesional TA activity can be explained by the fact that this muscle depends more strongly on motor cortex input ${ }^{59,60}$ which can be influenced by lesions in the internal capsule via corticospinal tract affections The role of the SOL muscle in mediating the interlimb relation in stroke subjects can be justified by its higher dependence on sensory input in relation to supraspinal control ${ }^{61,62}$. However, results also demonstrated that the functional influence of the ipsilesional SOL muscle over the contralesional limb depends on its role in the task. When this muscle acts as an agonist for movement (during terminal stance it promotes forward progression of the trunk ${ }^{53}$ ) it exerts an influence over the contralesional homolog muscle similar to the healthy subjects ${ }^{9}$. However, when its activity is more related to postural control, like during loading response, it exerts a biomechanically disadvantageous influence over the contralesional limb, considering the double inverted pendulum model and the interlimb relation observed in healthy subjects ${ }^{9}$. These findings support the argument for the dysfunction of the ventral-medial system over the ipsilesional limb, also hypothesised in other studies ${ }^{30-32}$, as one of the causes for impaired interlimb relation in stroke subjects. This hypothesis assumes special relevance considering that the stroke participants in this study present lesions in the internal capsule, which can be associated to dysfunction of the cortico-reticular pathway ${ }^{63}$, responsible for ipsilateral postural control.

According to Bowden et al ${ }^{49}$, 2006, the propulsive impulse provides a quantitative measure of the coordinated output of both lower limbs in stroke patients. The relative propulsive impulse of the contralesional limb was lower than the observed 
in the control group $(\approx 40 \%)$, which means that the ipsilesional limb energy loss during initial contact and loading response probably may not be compensated by propulsion of the contralesional limb ${ }^{5,7}$. The contralesional limb of stroke subjects has been shown to produce significantly less mechanical work output than of the healthy subjects ${ }^{64}$. The relative propulsive impulse of the ipsilesional limb exceeds the braking impulse $(\approx$ $60 \%$ ) of the contralesional limb during initial contact and loading response and probably accelerates the centre of mass ${ }^{5,7}$. It is important to note that in the control group, this propulsive impulse of the TRAIL was also higher than 50\%. In fact, previous studies on healthy subjects demonstrate that the positive mechanical work of the TRAIL during DS exceeds the negative mechanical work performed by the LEAD ${ }^{9}$.

\section{Study limitations}

The relationship between muscle activity and mechanical output depends on a number of nonlinear intrinsic properties; that is, on force-length-velocity relations that make the relationship difficult to predict. Given this limitation, it would be important to analyse the relation of joint moment power between limbs during DS and between the propulsive and braking impulse of each limb. This knowledge would help understanding the role of each muscle in both lower limbs of stroke subjects on gait mechanical output during step-to-step transition.

\section{CONCLUSIONS}

The results obtained in this study as to the propulsive impulse demonstrate that the contralesional limb presents lower performance in forward propulsion when compared with the ipsilesional limb and the control group. Despite exerting an indirect functional influence over the activity of plantar flexors, the ipsilesional limb exerted a dysfunctional influence during initial contact and loading response over the contralesional limb during pre-swing. The influence of the ipsilesional limb over the contralesional limb was classified based on the interlimb relation observed in healthy subjects ${ }^{9}$, the double-inverted pendulum model ${ }^{5,7}$ and the role of individual muscles during the stance phases associated to step-to-step transition ${ }^{38,53}$. These findings suggest that the lower performance of the contralesional limb in forward propulsion is not only related to contralateral supraspinal damage, but also to the influence of the ipsilesional limb in stance subphases of increased postural control demand. 
The results here present arguments for considering an indirect impact of a postural control dysfunction of the ipsilesional limb on the performance and efficiency of gait in stroke subjects. Future works should be developed to explore this possibility as the lower performance and lower efficiency of gait in subjects with stroke has been attributed mainly to alterations in the contralesional limb.

Considering the above, rehabilitation strategies should pay special attention to the ipsilesional limb to potentiate the contralesional limb activity in subjects with stroke affecting the subcortical structures in the territory of medial cerebral artery, such as in the internal capsule. Specifically, the results obtained suggest that improving postural control of the ipsilesional limb could have positive effects over the interlimb relation during step-to-step transition and consequently, over the walking performance.

\section{References}

1. Keenan MA, Perry J, Jordan C. Factors affecting balance and ambulation following stroke. Clinical Orthopaedics and Related Research 1984; 182:165-71.

2. Olney SJ, Richards C. Hemiparetic gait following stroke. Part I: Characteristics. Gait \& Posture 1996; 4:136-48.

3. Detrembleur C, Dierick F, Stoquart G, Chantraine F, Lejeune T. Energy cost, mechanical work, and efficiency of hemiparetic walking. Gait \& Posture 2003; 18:47-55.

4. Donelan JM, Kram R, Kuo AD. Mechanical work for step-to-step transition is a major determinant of the metabolic cost. Journal of Experimental Biology 2002; 205:3717-27.

5. Kuo $A D$, Donelan $M$, Ruina $A$. The six determinants of gait in the inverted pendulum analogy: a dynamic walking perspective. Human Movement Science 2007; 26:617-56.

6. Kuo AD. Energetics of actively powered locomotion using the simplest walking model. Journal of Biomechanical Engineering 2002; 124:113-20.

7. Kuo $A D$, Donelan $M$, Ruina $A$. Energetic consequences of walking like an inverted pendulum: step-to-step transitions. Exercise Sports Science Review 2005; 33:88-97.

8. Donelan J, Kram R, Kuo A. Mechanical work for step-to-step transitions is a major determinant of the metabolic cost of human walking. The Journal of Experimental Biology 2002; 205:3717-27.

9. Sousa ASP, Silva A, Tavares JMRS. Interlimb relation during the double support phase of gait: an electromyographic, mechanical and energy based analysis. Proceedings of the Institution of Mechanical Engineers, Part H: Journal of Engineering in Medicine 2012; 227.

10. Sousa ASP, Santos R, Oliveira FPM, Carvalho P, Tavares JMRS. Analysis of ground reaction force and electromyographic activity of the gastrocnemius muscle during double support. Proceedings of the Institution of Mechanical Engineers, Part H: Journal of Engineering in Medicine 2012; 226:397-405.

11. Corna S, Galante M, Grasso M, Nardone A, Schieppati M. Unilateral displacement of lower limb evokes bilateral EMG responses in leg and foot muscles in standing humans. Experimental Brain Research 1996; 109:83-91.

12. Dietz V. Human neuronal control of automatic functional movements: interaction between central programs and afferent input. Physiological Reviews 1992; 72:33-69.

13. Bajwa S, Edgley SA, Harrison PJ. Crossed actions on group II-activated interneurones in the midlumbar segments of the cat spinal cord. The Journal of Physiology 1992; 455:205-17. 
14. Davies HE, Edgley SA. Inputs to group II-activated midlumbar interneurones from descending motor pathways in the cat. The Journal of Physiology 1994; 479:463-73.

15. Dietz V, Berger W. Interlimb coordination of posture in patients with spastic paresis. Brain 1984; 107:965-78.

16. Roerdink M, Lamoth CJ, Kwakkel G, van Wieringen PC, Beek PJ. Gait Coordination After Stroke: Benefits of Acoustically Paced Treadmill Walking. Physical Therapy 2007; 87:1009-22.

17. Wu C-Y, Chou S-H, Chen C-L, Kuo M-Y, Lu T-W, Fu Y-C. Kinematic analysis of a functional and sequential bimanual task in patients with left hemiparesis: intra-limb and interlimb coordination. Disability and Rehabilitation 2009; 31:958-66.

18. Crone C, Johnsen LL, Biering-Sørensen F, Nielsen JB. Appearance of reciprocal facilitation of ankle extensors from ankle flexors in patients with stroke or spinal cord injury. Brain 2003; 126:495-507.

19. Marque $\mathrm{P}$, Simonetta-Moreau M, Maupas E, Roques CF. Facilitation of transmission in heteronymous group II pathways in spastic hemiplegic patients. Journal of Neurology, Neurosurgery \& Psychiatry 2001; 70:36-42.

20. Maupas E, Marque P, Roques CF, Simonetta-Moreau M. Modulation of the transmission in group II heteronymous pathways by tizanidine in spastic hemiplegic patients. Journal of Neurology, Neurosurgery \& Psychiatry 2004; 75:130-5.

21. Nardone A, Schieppati M. Reflex contribution of spindle group la and II afferent input to leg muscle spasticity as revealed by tendon vibration in hemiparesis. Clinical Neurophysiology 2005; 116:1370-81.

22. Matsuyama K, Mori F, Nakajima K, Drew T, Aoki M, Mori S. Locomotor role of the corticoreticular-reticulospinal-spinal interneuronal system. In: Shigemi Mori DGS, Mario W, editors. Progress in Brain Research: Elsevier; 2004. p. 239-49.

23. Jankowska E, Hammar I, Slawinska U, Maleszak K, Edgley SA. Neuronal basis of crossed actions from the reticular formation on feline hindlimb motoneurons. The Journal of neuroscience: the official journal of the Society for Neuroscience 2003; 23:1867-78.

24. Chen G, Patten C, Kothari DH, Zajac FE. Gait differences between individuals with poststroke hemiparesis and non-disabled controls at matched speeds. Gait \& Posture 2005; 22:516.

25. Chen G, Patten C. Joint moment work during the stance-to-swing transition in hemiparetic subjects. Journal of Biomechanics 2008; 41:877-83.

26. Peterson CL, Hall AL, Kautz SA, Neptune RR. Pre-swing deficits in forward propulsion, swing initiation and power generation by individual muscles during hemiparetic walking. Journal of Biomechanics 2010; 43:2348-55.

27. Nadeau S, Gravel D, Arsenault AB, Bourbonnais D. Plantarflexor weakness as a limiting factor of gait speed in stroke subjects and the compensating role of hip flexors. Clinical Biomechanics 1999; 14:125-35.

28. Stoquart $G$, Detrembleur $C$, Lejeune TM. The reasons why stroke patients expend so much energy to walk slowly. Gait \& Posture 2012; 36:409-13.

29. Lamontagne A, Malouin F, Richards CL, Dumas F. Mechanisms of disturbed motor control in ankle weakness during gait after stroke. Gait \& Posture 2002; 15:244-55.

30. Silva A, Sousa ASP, Tavares J, Tinoco A, Santos R, Sousa F. Ankle dynamic in stroke patients. Agonist vs antagonist muscle relations. Somatosensory and Motor Research 2012; 29:111-6.

31. Silva A, Sousa ASP, Pinheiro R, Ferraz J, Tavares JMRS, Santos R, et al. Activation timing of soleus and tibialis anterior muscles during sit-to-stand and stand-to-sit in post-stroke vs healthy subjects. Somatossensory and Motor Research 2012; 30:48-85.

32. Silva A, Sousa ASP, Pinheiro R, Tavares JMRS, Santos R, Sousa F. Soleus activity in poststroke subjects: movement sequence from standing to sitting. Somatossensory and Motor Research 2012; 29:71-6. 
33. Shiavi R, Bugle H, Limbird T. Electromyographic gait assessment, Part 2: Preliminary assessment of hemiparetic synergy patterns. J Rehabil Res Dev 1987; 24:24 - 30.

34. Kautz SA, Patten C. Interlimb influences on paretic leg function in post-stroke hemiparesis. Journal of Neurophysiology 2005; 93:2460-73.

35. Tseng S-C, Morton SM. Impaired Interlimb Coordination of Voluntary Leg Movements in Poststroke Hemiparesis. Journal of Neurophysiology 2010; 104:248-57.

36. Lamontagne A, Malouin F, Richards CL. Locomotor-specific measure of spasticity of plantarflexor muscles after stroke. Archives of Physical Medicine and Rehabilitation 2001; 82:1696-704.

37. Lamontagne A, Richards CL, Malouin F. Coactivation during gait as an adaptive behavior after stroke. Journal of Electromyography and Kinesiology 2000; 10:407-15.

38. Neptune R, Kautz S, Zajac F. Muscle force redistributes segmental power for body progression during walking. Gait \& Posture 2004; 19:194-205.

39. Zajac FE, Neptune RR, Kautz SA. Biomechanics and muscle coordination of human walking: Part II: Lessons from dynamical simulations and clinical implications. Gait \& Posture 2003; 17:1-17.

40. Whitle M. Gait Analysis: An Introduction. $4^{\text {th }}$ ed. USA: Elsevier; 2007.

41. Sousa ASP, Tavares JMRS. Effect of gait speed on muscle activity patterns and magnitude during stance. Motor Control 2012; 16:480-92.

42. Sekiya $\mathrm{N}$, Nagasaki $\mathrm{H}$, Ito $\mathrm{H}$, Furuna T. Optimal walking in terms of variability in step length. Journal of Orthopaedic and Sports Physical Therapy 1997; 26:266-72.

43. Shiavi R, Bugle H, Limbird T. Electromyographic gait assessment, part 1: Adult EMG profiles and walking speed. Journal of Rehabilitation Research and Development 1987; 24:1323.

44. Masani K, Kousaki M, Fukunaga T. Variability of ground reaction forces during treadmill walking. Journal of Applied Physiology 2002; 92:1885-90.

45. Milot M-H, Nadeau S, Gravel D. Muscular utilization of the plantarflexors, hip flexors and extensors in persons with hemiparesis walking at self-selected and maximal speeds. Journal of Electromyography and Kinesiology 2007; 17:184-93.

46. Requião LF, Nadeau S, Milot MH, Gravel D, Bourbonnais D, Gagnon D. Quantification of level of effort at the plantarflexors and hip extensors and flexor muscles in healthy subjects walking at different cadences. Journal of Electromyography and Kinesiology 2005; 15:393-405.

47. Mullineaux D, Bartlett $R$, Bennett $S$. Research methods and statistics in biomechanics and motor control. Journal of Sports Sciences 2001; 19:739-60.

48. Winter A. Biomechanics and Motor Control of Human Movement. 2nd ed. New York: Wiley; 1990.

49. Bowden MG, Balasubramanian CK, Neptune RR, Kautz SA. Anterior-posterior ground reaction forces as a measure of paretic leg contribution in hemiparetic walking. Stroke 2006; 37:872-6.

50. Yang JF, Winter DA. Electromyographic amplitude normalization methods: Improving their sensitivity as diagnostic tools in gait analysis. Archives of Physical Therapy Medicine and Rehabilitation 1984; 65:517-21.

51. Perry J, Garrett M, Gronley JK, Mulroy SJ. Classification of walking handicap in the stroke population. Stroke 1995; 26:982-9.

52. Stubbs P, Sinkjaer T, Nielsen J, Nielsen J, Mrachacz-Kersting N. Evidence of spinal cord mediation of interlimb coordination in the human soleus muscle. 7th edition of Progress in Motor Control; France2009.

53. Neptune R, Kautz A, Zajac E. Contributions of the individual ankle flexors to support, forward progression and swing initiation during normal walking. Journal of Biomechanics 2001; 34:1387-98. 
54. Sadeghi H, Allard P, Barbier F, Sadeghi S, Hinse S, Perrault R, et al. Main functional roles of knee flexors/extensors in able-bodied gait using principal component analysis (I). The Knee 2002; 9:47-53.

55. Liu M, Anderson F, Pandy M, Delp S. Muscles that support the body also modulate forward progression during walking. Journal of Biomechanics 2006; 39:2623-30.

56. McGowan CP, Neptune RR, Kram R. Independent effects of weight and mass on plantar flexor activity during walking: implications for their contributions to body support and forward propulsion. Journal of Applied Physiology 2008; 105:486-94.

57. Turns LJ, Neptune RR, Kautz SA. Relationships between muscle activity and anteroposterior ground reaction forces in hemiparetic walking. Archives of Physical Medicine and Rehabilitation 2007; 88:1127-35.

58. Achache V, Mazevet D, Iglesias C, Lackmy A, Nielsen J, Katz R, et al. Enhanced spinal excitation from ankle flexors to knee extensors during walking in stroke patients. Clinical Neurophysiology 2010; 121:930 - 8.

59. Yang JF, Gorassini M. Spinal and brain control of human walking: implications for retraining of walking. The Neuroscientist 2006; 12:379-89.

60. Capaday C, Lavoie BA, Barbeau H, Schneider C, Bonnard M. Studies on the corticospinal control of human walking. I. Responses to focal transcranial magnetic stimulation of the motor cortex. Journal of Neurophysiology 1999; 81:129-39.

61. Beres-Jones JA, Harkema SJ. The human spinal cord interprets velocity-dependent afferent input during stepping. Brain 2004; 127:2232-46.

62. Harkema SJ, Hurley SL, Patel UK, Requejo PS, Dobkin BH, Edgerton VR. Human lumbosacral spinal cord interprets loading during stepping. Journal of Neurophysiology 1997; 77:797-811.

63. Drew T, Prentice S, Schepens B. Cortical and brainstem control of locomotion. In: Shigemi Mori DGS, Mario W, editors. Progress in Brain Research: Elsevier; 2004. p. 251-61.

64. Brown DA, Kautz SA. Speed-dependent reductions of force output in people with poststroke hemiparesis. Physical Therapy 1999; 79:919-30.

\section{Suppliers}

a. Bertec Corporation 6171 Huntley Road Suite J Columbus, OH 43229 U.S.A.

b. PLUX wireless biosignals S.A. Av. 5 de Outubro, 70 - 8, 1050-059 Lisboa, PORTUGAL.

c. Brower Timing Systems 12660 South Fort Street Suite 102 Draper, Utah 84020 USA.

d. Noraxon U.S.A. Inc., 15770 North Greenway-Hayden Loop, Suite 100 Scottsdale, AZ 85260.

c. BIOPAC Systems, Inc., 42 Aero Camino Goleta, CA 93117 USA. 


\section{FIGURE CAPTIONS}

Figure 1: The intervals used to assess the interlimb relation during the stance phase of walking in stroke subjects were defined using the variation of the ground reaction force. The intervals are shown for each series: a) when the ipsilesional limb was the TRAIL limb and the contralesional limb was the LEAD limb, and b) when the ipsilesional limb was the LEAD limb and the contralesional limb was the TRAIL limb.

Figure 2: Representation of statistically significant correlations between the limbs found in the stroke group. Statistically significant correlations occurred in EMG activity between the paretic TRAIL limb and the non-paretic LEAD limb during DS (a), and between the non-paretic TRAIL limb during TS and the paretic LEAD limb during DS (b) and (c) (*significant correlation $(\mathrm{p}<0.05))$.

Figure 3: Mean (bars) and standard deviation (error bars) of propulsive and braking impulse observed in paretic and non-paretic limbs of subjects with stroke and healthy controls during DS and TS (*significant correlation $\left(\mathrm{p}^{<0.05}\right)$ ).

Figure 4: Mean (bars) and standard deviation (error bars) values of the percentage of TRAIL propulsive contribution of the paretic and non-paretic limbs of subjects with stroke and of TRAIL propulsion contribution in healthy controls during DS (*significant correlation $(\mathrm{p}<0.05)$ ). 


\section{FIGURES}

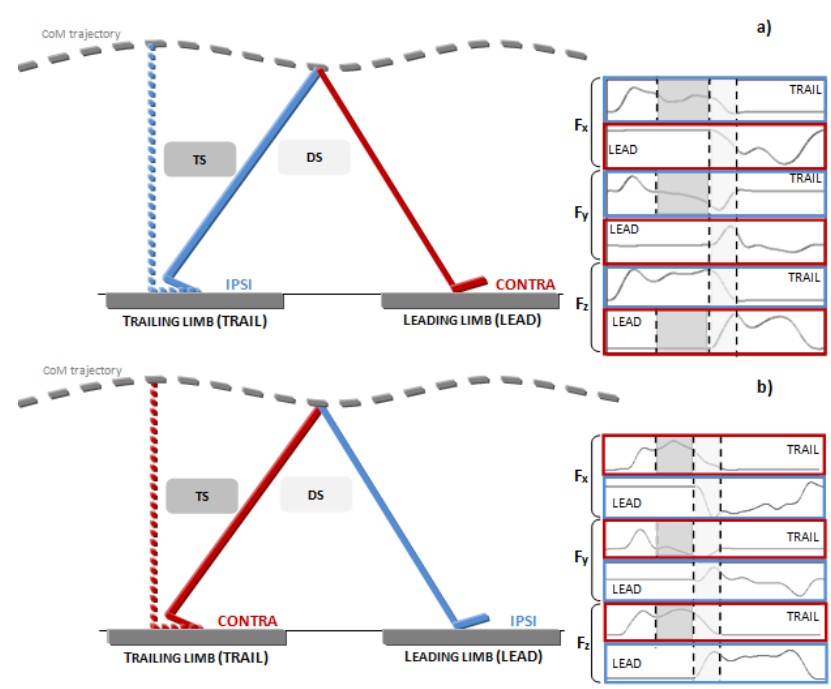

Figure 1
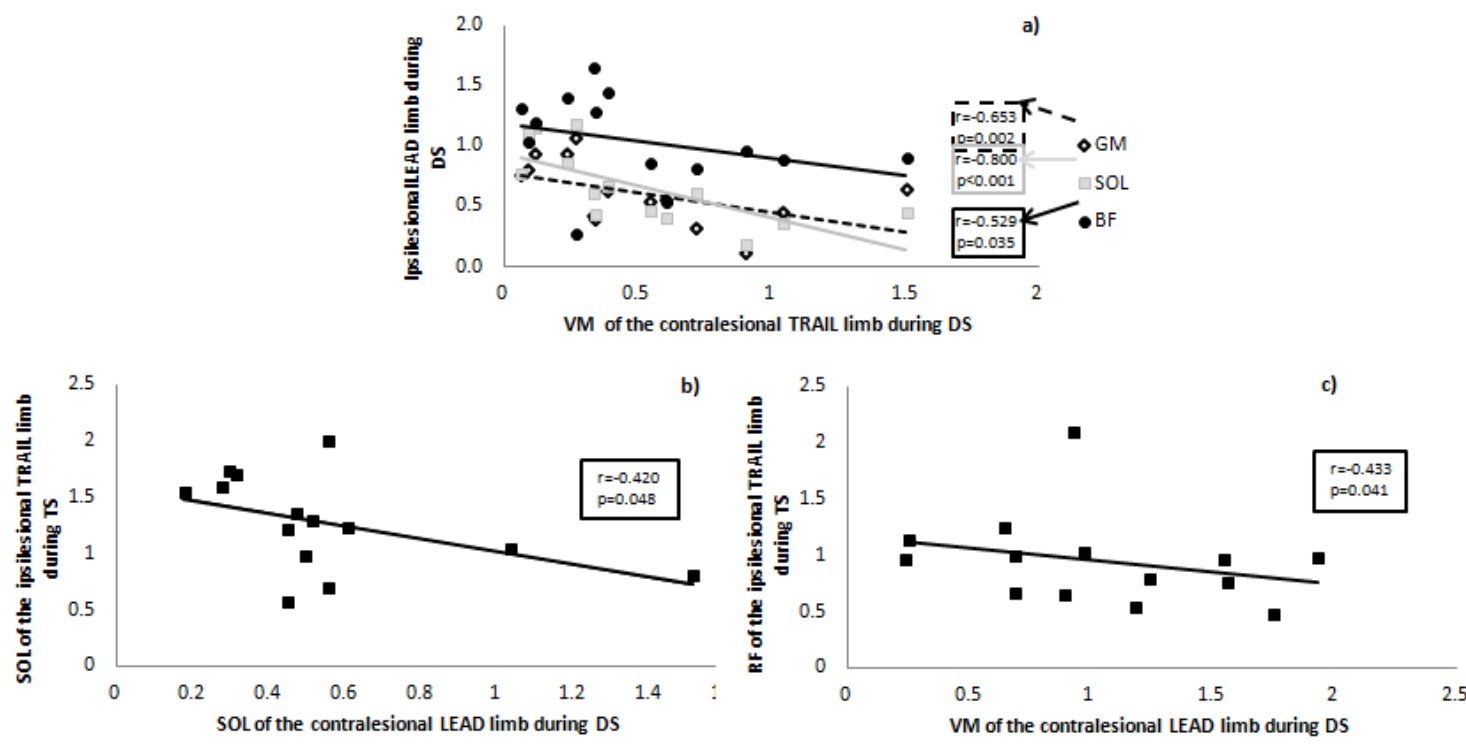

Figure 2 


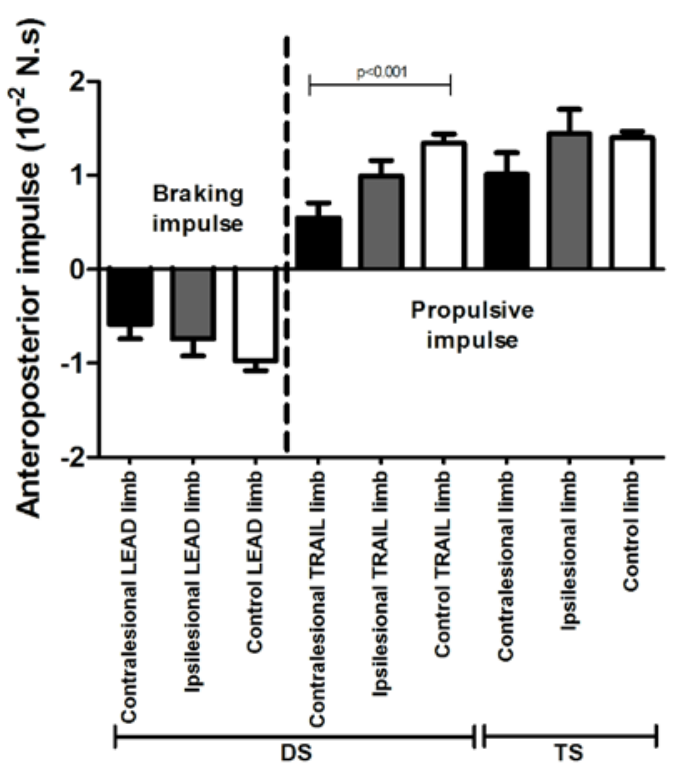

Figure 3

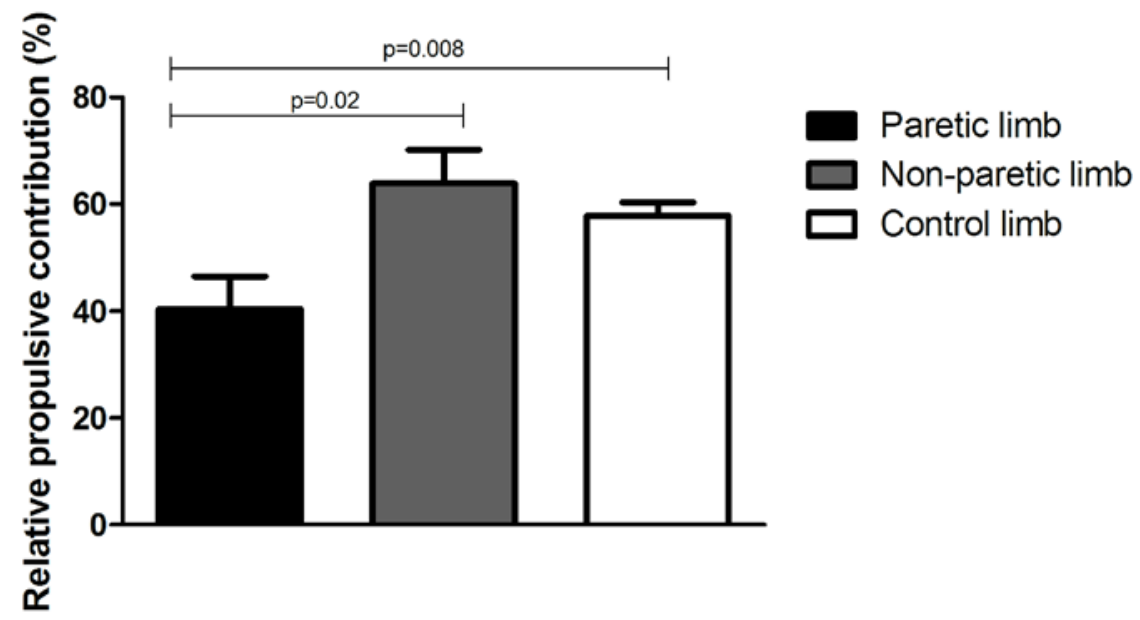

Figure 4 


\section{TABLE CAPTIONS}

Table 1: Mean and standard deviation (SD) values of age, height and weight of the healthy and stroke groups, and the average values of the self-selected walking speeds adopted by each group. 
TABLES

Table 1

\begin{tabular}{cccc}
\hline \multirow{2}{*}{ Variables } & Stroke group & Control group & \\
\cline { 2 - 3 } & Mean \pm SD & Mean \pm SD & \\
\hline Age (years) & $53.87 \pm 7.17$ & $49.24 \pm 7.69$ & 0.070 \\
\hline Height (m) & $1.65 \pm 0.10$ & $1.66 \pm 0.09$ & 0.942 \\
\hline Body weight $(\mathrm{Kg})$ & $75.29 \pm 7.03$ & $67.40 \pm 8.76$ & 0.006 \\
\hline Self-selected gait speed $\left({\left.\mathrm{m} . \mathrm{s}^{-1}\right)}\right.$ & $0.57 \pm 0.13$ & $1.00 \pm 0.03$ & $<0.001$ \\
\hline
\end{tabular}

